\title{
Parametric Optimization and Analysis of Adaptive Equalization Algorithms for Noisy Speech Signals
}

\author{
G.R. Mishra, Mudit Shukla*, O. P. Singh, Sachin Kumar, Kamal Ahmad \\ Department of Electronics \& Communication Engineering, Amity School of Engineering \& Technology \\ Amity University Uttar Pradesh, Lucknow Campus, India
}

\begin{abstract}
Adaptive equalization technique plays key role in modern digital communication systems. Adaptive digital filters are widely used in the area of signal processing such as echo cancellation, noise cancellation, channel equalization and beamforming. This paper presents the performance analysis of RLS and CMA algorithm in presence of noisy audio signal. The analysis shows that in CMA algorithm the convergence rate is low as compare to RLS algorithm whereas CMA algorithm requires low computing power and relatively better performance. The parameters of proposed equalizer have been optimized and simulated using Simulink.

Index Terms-CMA, Equalization, Noisy audio signal, RLS algorithms
\end{abstract}

\section{Introduction}

In the digital communication system, when the signal passes through a channel, signal gets distorted due to various causes. One of the most common causes is Inter symbol interference (ISI). In presence of ISI, receiver does not clearly understand the received samples. The linear distortion which is produced by the channel is minimized by the most important part of the receiver called equalizer by a process called equalization. Equalization is defined as process of change in the frequency of a signal in order to maintain the signal at the normal level [1]. If a signal is transmitted through a channel, its frequency can vary. So an equalizer is most essential element of a receiver system, which removes emphasized frequency from the transmitted signal [2].

If the characteristics of the channel are known than optimum setting for equalizer can be computed. But in the practical system the characteristics of the channel is the big problem, so adaptive equalizers are used. Adaptive filter can be used in the system for different purposes such as system identification, noise cancellation, echo cancellation and equalization. When adaptive filter theory converges with the equalizer then we get an adaptive equalizer. Adaptive equalizer has capability to change the coefficients of transfer function as per system requirement [3].

The adaptive equalization is of two types: trained equalization and blind equalization.

\section{A) Trained Equalization}

Trained equalization is based on the pseudo random sequence. Which is the random pattern of bits consists of ones and zeros called training sequence known both to the transmitter and receiver. In this paper we use two types of trained equalization algorithms named LMS and RLS.

Least Mean Square (LMS) Algorithm: The LMS algorithm is a stochastic gradient algorithm, which means that the gradient of the error performance surface with respect to the free parameter vector changes randomly from one iteration to the next. The LMS algorithm achieves simplicity of implementation by using instantaneous estimates of the autocorrelation matrix of the input signal vector, and the cross-correlation vector between the input vector and the desired response. The LMS algorithm has two major shortcomings: slow rate of convergence, and sensitivity to the eigen value spread (i.e., the ratio of the largest eigen value to the smallest eigen value) of the correlation matrix of the input signal vector [4].

Recursive Least Square $(R L S)$ : The RLS algorithm utilizes continuously updated estimates of autocorrelation matrix of the input signal vector, and the cross-correlation vector between the input vector and the desired response quantities, which go back to the beginning of the adaptive process. Accordingly, the RLS algorithm exhibits the following properties:

- Rate of convergence that is typically an order of magnitude faster than the LMS algorithm.

- Rate of convergence that is invariant to the eigen value spread of the correlation matrix of the input Vector [4].

\section{B) Blind Equalization}

In the blind equalization no training sequence is used. However blind equalization is capable to compensate amplitude and delay distortion of a communication channel. The channel equalization performed without help of training sequence. The term blind indicate that the equalization is performed blindly on the data without a reference signal. The blind equalization depends on the knowledge of signal's structure and its 
statistical properties. One of the most important advantages of the blind equalization is that no bandwidth is wasted by its transmission because no training sequence is used at the start up [5]. The algorithm which we use in this paper of blind equalization is Godard or constant modulus algorithm (CMA).

Godard or Constant Modulus Algorithm (CMA): Godard developed algorithm for the complex valued signal is the most popular algorithm for the blind equalization of QAM signal is called the constant modulus algorithm [6]. CMA tries to minimize the constant modulus cost function $\mathrm{J}_{\mathrm{CM}}$. Consider a input data symbol $\left\{\mathrm{a}_{\mathrm{k}}\right\}$. The squared magnitude of received sample $z(n)$ and Godard dispersion constant $R_{2}$ depends only on the $\left\{a_{k}\right\}$. CMA minimizes the difference between these two by adjusting the weights of the equalizer. This gives the value of $\mathrm{J}_{\mathrm{CM}}$.

$$
\mathrm{J}_{\mathrm{CM}}=\mathrm{E}\left[\left(\mathrm{abs}(\mathrm{z}(\mathrm{n}))^{2}-\mathrm{R}_{2}\right)^{2}\right]
$$

Where $z(n)$ is the equalizer output at time $n$. The equalizer coefficient update equalization in CMA uses a gradient descent to minimize $\mathrm{J}_{\mathrm{CM}}$. The equation is given by Godard as-

For determining the value of $\mathrm{R}_{2}$

$$
\mathrm{C}(\mathrm{n}+1)=\mathrm{C}(\mathrm{n})-\mu \mathrm{y}(\mathrm{n}) \mathrm{z}(\mathrm{n})\left[\left(\operatorname{abs}(\mathrm{z}(\mathrm{n}))^{2}-\mathrm{R}_{2}\right)\right]
$$

$$
\mathrm{R}_{2}=\mathrm{E}\left[\mathrm{a}(\mathrm{n})^{4}\right] / \mathrm{E}\left[\mathrm{abs}(\mathrm{a}(\mathrm{n}))^{2}\right]
$$

Where $\mathrm{a}(\mathrm{n})$ is the signal to be transmitted and $\mathrm{E}[\mathrm{is}$ is the exception over all possible transmitted data sequence. In this paper we use three adaptive algorithms. Out of these three algorithms, two algorithms are based on trained equalization LMS and RLS. The LMS algorithm is taken as a reference algorithm during this work. One blind equalization algorithm CMA is used [7]. We evaluate the performance of the adaptive equalizer in the presence of noisy audio source on the basis of their mean square error (MSE) convergence rate.

\section{Proposed Adaptive Equalizer Combined With Noisy Audio Source}

Fig 1 shows the proposed block diagram of the adaptive equalizer combined with the noisy audio source. At the transmitter side the noise is added with the audio signal and then signal is encoded by the help of differential encoder. Encoded data is modulated using QAM modulation scheme and BPSK is considered as symbol constellation. After that this modulated data is transmitted through an AWGN channel. The signal is received by a receiver having an equalizer. Three adaptive algorithms are performed simultaneously to make the equalizer adaptive and evaluate the performance of the adaptive equalizer in the term of mean square error convergence rate.

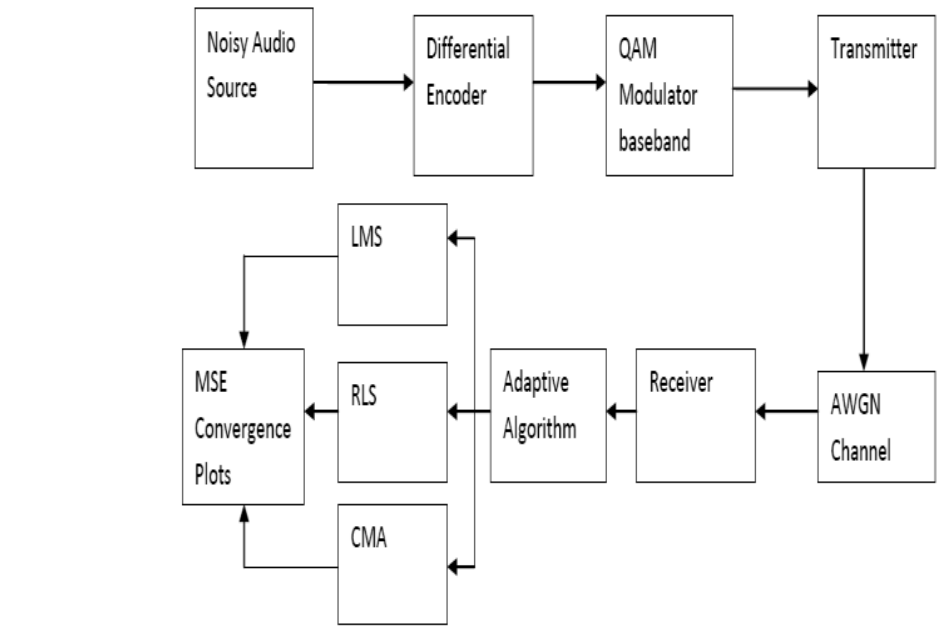

Fig 1- Simulink Diagram of Proposed Adaptive Equalizer Combined With Noisy Audio Source

\section{Simulation Parameters}

The proposed model for the adaptive equalizer combined with the noisy audio source is simulated on the MATLAB10.0 Simulink. Table 1 shows the parameters and its corresponding value/technique consider during this work. The step size is taken as 0.01 for the LMS and CMA. A slight change in the step size can give other outputs. We consider a mono audio signal of $8000 \mathrm{~Hz}, 16-$ bit. The LMS and RLS is operated in the training/decision direct mode, we can switch between the modes during the simulation. 


\begin{tabular}{|l|l|}
\multicolumn{1}{|c|}{ Tarameter 1: Simulation Parameters } \\
\hline Audio Signal & \multicolumn{1}{|c|}{ Value/Technique } \\
\hline Signal Constellation & $8000 \mathrm{~Hz}, 16-$ bit, Audio mono \\
\hline Modulation & QPSK \\
\hline Channel & AWGN \\
\hline Signal to noise Ratio & $40 \mathrm{~dB}$ \\
\hline Number of Taps (LMS,RLS,CMA) & 2 \\
\hline Step Size (LMS,CMA) & 0.01 \\
\hline Leakage Factor (LMS,CMA) & 1 \\
\hline Forgetting Factor (RLS) & 0.9000 \\
\hline Initial Weights (LMS,RLS,CMA) & $0.6238,-0.1752$ \\
\hline Mode (LMS,RLS) & Training/Decision Direct \\
\hline
\end{tabular}

\section{Simulation Results}

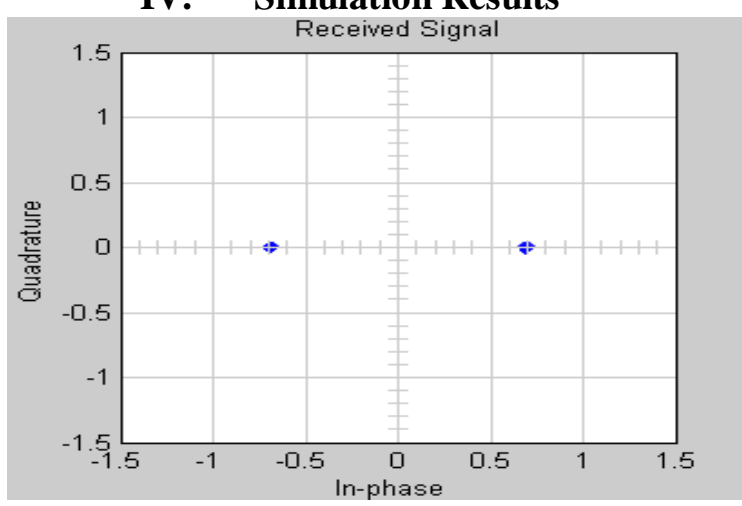

Fig 2- Received Signal at the Output of the Channel (RLS)

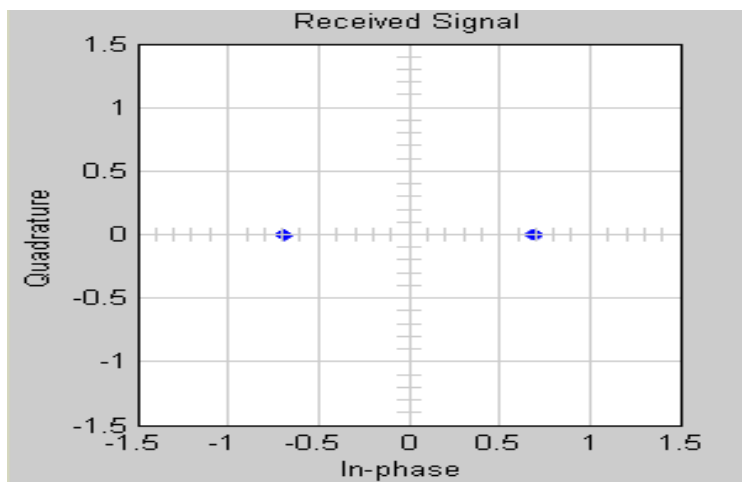

Fig 3-Received Signal at the Output of the Channel (CMA)

Fig 2 shows a scatter plot of the received signal at the output of the channel when the configurable algorithm is RLS. This shows that during implementation of RLS algorithm while the noisy audio signal passes through AWGN channel, the output is deviated from desired value. The simulation result of CMA algorithm is shown in Fig 3. This result shows scatter plot of the received signal at the output of the channel, which is similar to result of RLS. 


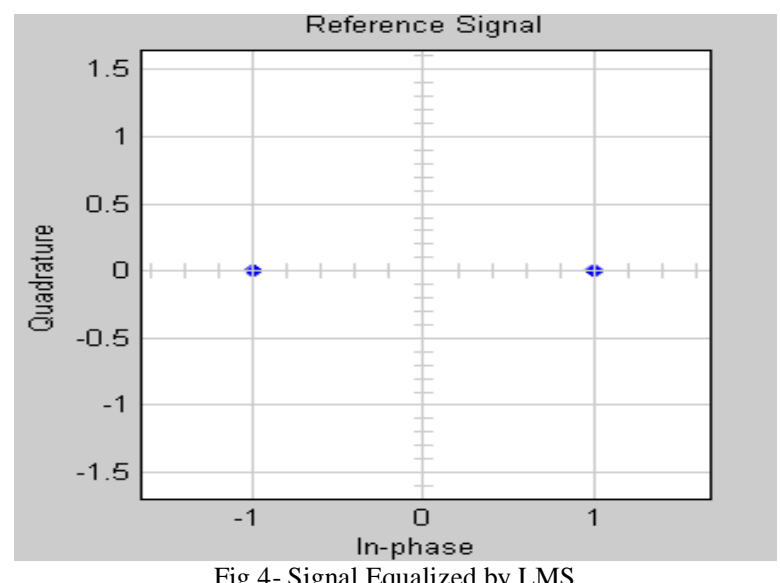

Fig 4 shows a scatter plot of the signal equalized by the reference equalizer. We consider LMS as a reference equalizer.

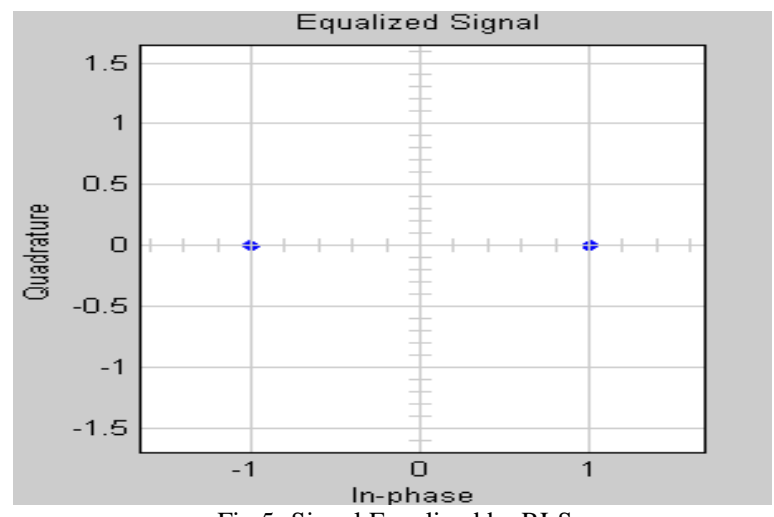

Fig 5- Signal Equalized by RLS

Fig 5 shows a scatter plot of the signal equalized by the RLS equalizer. This result is comparable to the reference signal (Shown in figure 4). It indicates that the performance of RLS algorithm in presence of noisy speech signal.

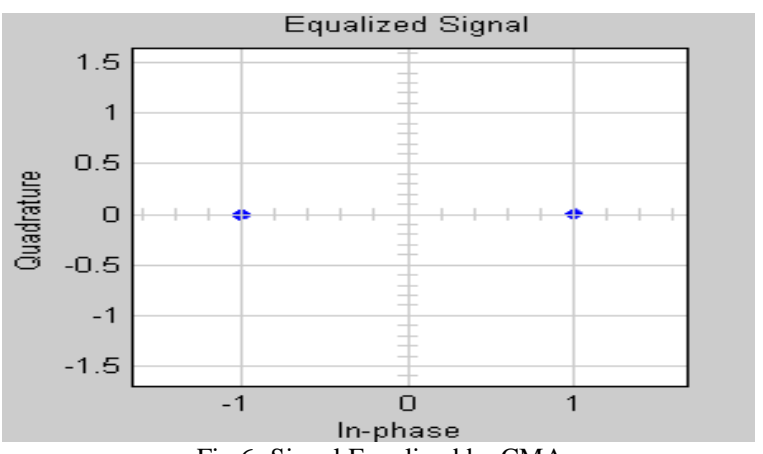

Fig 6- Signal Equalized by CMA

Fig 6 shows a scatter plot of the signal equalized by the CMA equalizer. This result is also similar to the reference signal (Shown in figure 4). These results show that the signal equalization capabilities of both algorithms are similar.

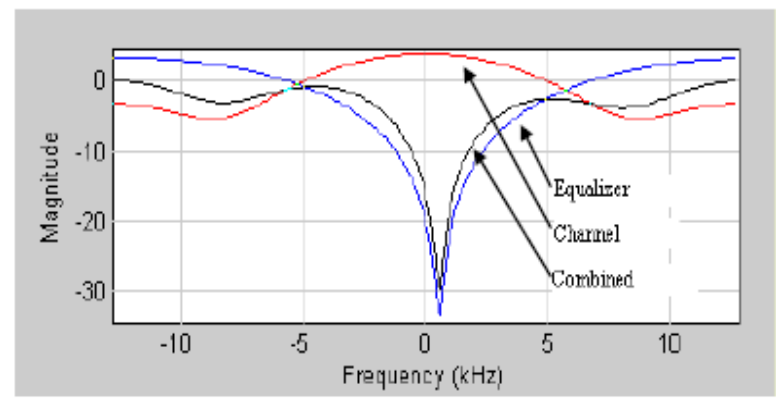

Fig 7- Frequency Response of Equalizer (RLS), Channel and

Combined 


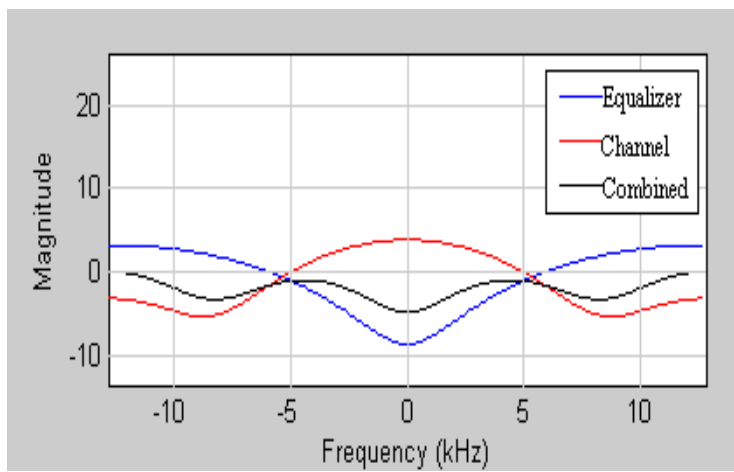

Fig 8- Frequency Response of Equalizer (CMA), Channel and Combined

Fig 7 and 8 shows the frequency response of the equalizer (RLS and CMA), channel and combined. We can see that in the presence of noisy audio source RLS equalizer has very poor frequency response as compare to the CMA equalizer. The frequency response of the combination of channel and CMA equalizer is approximately desired, which shows that in the case of noisy audio source the CMA equalizer has better frequency response.

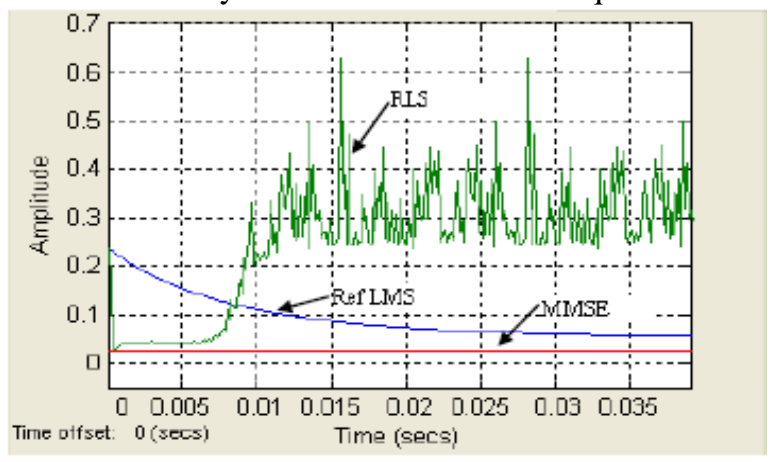

Fig 9- MSE Convergence Plot for RLS

Fig 9 shows the MSE convergence plot of the RLS equalizer. We can see that the convergence rate of RLS equalizer is very much fast, almost in $0.001 \mathrm{sec}$ it converges from 0.24 towards the minimum mean square error (MMSE) but for a very short period of time 0.008 . After $0.008 \mathrm{Sec}$ the RLS equalizer not succeeds to achieve that convergence rate.

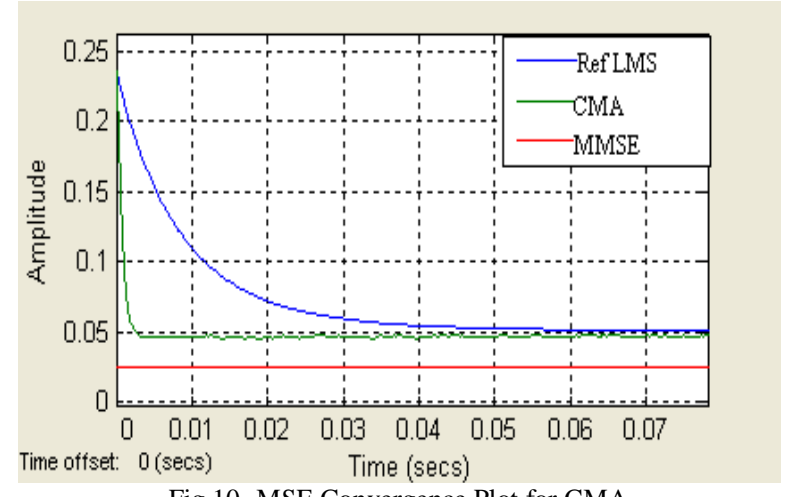

Fig 10- MSE Convergence Plot for CMA

Fig 10 shows the MSE convergence plot of the CMA equalizer. We can see that the CMA equalizer converges from 0.24 to 0.05 in almost $0.05 \mathrm{sec}$. Although it is slower than RLS but successfully maintain the convergence rate. On the other hand the LMS equalizer takes time to converge [8, 15]. During the simulation process various values of the step size have been taken and the desired results have been found at optimum value of 0.01 . MSE is one of the important parameter which decides the performance of equalizer. The simulation result shows that CMA has better MSE convergence as comparison to RLS. Since CMA is blind equalization algorithm and RLS is trained equalization algorithm so we can conclude that performance of blind equalization algorithm is better as compare to trained equalization algorithm in presence of noisy audio signal.

\section{Conclusion}

Thus using MATLAB simulation performance parameters of the LMS, RLS and CMA are optimized and analyzed in the presence of the noisy audio source. It is analyzed that the step size $(\mu)$ is the important parameter of these algorithms and the convergence characteristics decidedly depends on the $\mu$. So the value of $\mu$ can affects the convergence rate and mean square error. Also it is examine that in the presence of noisy audio 
source, trained equalization algorithm RLS gives faster convergence rate as compare to the LMS, although needing higher computational capability. The obtained results show that the CMA equalizer is very promising in presence of noisy audio source. However CMA equalizer gives low convergence rate, comparable to that of the RLS equalizer but has better performance and requires no much computing power.

\section{References}

[1] John G. Proakis, "Digital Communications" McGraw-Hill International Edition, Fourth Edition 2001.

[2] Theodore S. Rappaport, "Wireles Communications principles \& practice Article”, Second Edition.2002

[3] Shahid U.H.Qureshi, “Adaptive Equalization”, Vol-73, Issue-9, IEEE Proceedings-Sep 1985

[4] S. Haykin, Adaptive Filter Theory, Prentice Hall, Englewood Cliffs, NJ, 4th edition, 2002.

[5] C. R. Johnson et al., "Blind equalization using the constant modulus criterion: A review," Proc. IEEE, vol. 86, no. 10, pp.19271950, Oct. 1998.

[6] Domnique N. Godard, "Self-Recovering Equalization and carrier tracking in Two-Dimensional Data Communication Systems", IEEE transactions on Communications, vol. COM-28, No.11, Nov.1980

[7] E. Kalpana, O. Uma Maheswari, "Architecture Design for an Adaptive Equalizer", National Conference, Pune..

[8] O. Dabeer, "Convergence analysis of the LMS and the constant modulus algorithms," Ph.D. dissertation, Univ. Calif., San Diego, La Jolla, 2002.

[9] G. UNGERBOECK, "Theory on the speed of Convergence in adaptive Equalizers for digital Communications." IBM J. Res. Develop., Vol. 11, pp 546-555, 1972.

[10] V. WEERACKODY, S. A. KASSAM, and K. R. LAKER, "Convergence Analysis of an Algorithm for Blind Equalization, " IEEE TRANS. COMMUN., Vol. 39, pp. 856-865, June 1991.

[11] R. CUSANI and A. LAURENTI, "Convergence Analysis of CMA Blind Equalizer," IEEE Transac. Commun., Vol. 43, pp. 1304$1307,1995$.

[12] J. Yaun, K. Tsai, "Analyisis of the Multimodulus Blind Equalization Algorithm in QAM Communication Systems", IEEE Transactions on Communications, Vol. 53, No. 9, pp. 1427-1431, Sept. 2005.

[13] A. Beasley, A. Cole-Rhodes, "Performance of an Adaptive Blind Equalizer for QAM Signals", MILCOM 2005, Atlantic City, NJ, Oct. 2005.

[14] L. He, M. G. Amin, C. Reed, Jr., R. C. Malkemes, "A Hybrid Adaptive Blind Equalization Algorithm for QAM Signals in Wireless Communications”, IEEE Trans. on Signal Proc., Vol. 52, No. 7, pp. 2058-2069, July 2004

[15] Onkar Dabeer and Elias Masry "Convergence Analysis of the Constant Modulus Algorithm" IEEE TRANSACTIONS ON INFORMATION THEORY, VOL. 49, NO. 6, JUNE 2003 